\title{
OSCILLATORY PROPERTIES OF EVEN-ORDER ORDINARY DIFFERENTIAL EQUATIONS WITH VARIABLE COEFFICIENTS
}

\author{
OMAR BAZIGHIFAN AND ANDREA SCAPELLATO
}

Received 19 June, 2020

\begin{abstract}
The aim of this paper is to investigate oscillatory properties of even-order advanced differential equations. The key idea of our approach is to conduct a comparison with first order equations and use the Riccati transformation technique. Some new oscillation criteria are shown. Two examples are presented in order to clarify the main results.
\end{abstract}

2010 Mathematics Subject Classification: 34C10; 34K11

Keywords: oscillatory solutions, nonoscillatory solutions, advanced differential equations, Riccati transformation, comparison theorem

\section{INTRODUCTION}

Advanced differential equations can find application in several real world problems where the evolution rate depends not only on the present, but also on the future. Hence, an advance could be introduced into the equation in order to take into account the influence of potential future actions. For instance, it is worth pointing out that there are a lot of applications to dynamical systems, mathematics of networks, optimization, and their application in the mathematical modelling of engineering problems, such as concerning electrical power systems, materials, energy (see [13]).

In this paper, we establish new oscillation criteria for all solutions of non-linear even-order differential equations with variable coefficients and advanced term of the form

$$
\left(a(t)\left(w^{(n-1)}(t)\right)^{\beta}\right)^{\prime}+\sum_{i=1}^{j} q_{i}(t) g\left(w\left(\eta_{i}(t)\right)\right)=0, t \geq t_{0},
$$

where $j \geq 1$ and $\beta$ is a quotient of odd positive integers.

Throughout this paper, we suppose that:

- $a \in C^{1}\left(\left[t_{0}, \infty\right), \mathbb{R}\right)$,

- $a(t)>0, a^{\prime}(t) \geq 0, q_{i}, \eta_{i} \in C\left(\left[t_{0}, \infty\right), \mathbb{R}\right)$,

- $q_{i}(t) \geq 0$,

- $\eta_{i}(t) \geq t, \lim _{t \rightarrow \infty} \eta_{i}(t)=\infty, i=1,2, . ., j$, 
- $g \in C(\mathbb{R}, \mathbb{R})$ such that $\frac{g(x)}{x^{\beta}} \geq k>0$, for $x \neq 0$

- $\int_{t_{0}}^{\infty} \frac{1}{a^{1 / \beta}(s)} \mathrm{d} s=\infty$.

By a solution of (1.1) we mean a function $w \in C^{n-1}\left[t_{w}, \infty\right), t_{w} \geq t_{0}$, which has the property $a(t)\left(w^{(n-1)}(t)\right)^{\beta} \in C^{1}\left[t_{w}, \infty\right)$, and satisfies (1.1) on $\left[t_{w}, \infty\right)$. A solution of (1.1) is called oscillatory if it has arbitrarily large zeros on $\left[t_{w}, \infty\right)$; otherwise, it is called non-oscillatory. Equation (1.1) is said to be oscillatory if all of its solutions are oscillatory.

\section{THE AIM OF THIS PAPER}

In the last decade, the interest in studying of oscillation properties for differential equations increased (see for instance [3-8,10-12,14-19,23-25]). On the other hand, the study of qualitative properties of solutions to differential equations were extensively studied also in [20-22].

The aim of this paper is to complement and enrich the results contained in $[1,2,9]$.

For this reason, it is interesting to discuss briefly the aforementioned results.

By using the comparison technique, the equation

$$
\left(\left(w^{(n-1)}(t)\right)^{\beta}\right)^{\prime}+q(t) w^{\beta}(\eta(t))=0,
$$

have been studied by Agarwal and Grace [1] and they proved that (2.1) is oscillatory if

$$
\liminf _{t \rightarrow \infty} \int_{t}^{\eta(t)}(\eta(s)-s)^{n-2}\left(\int_{s}^{\infty} q(t) d t\right)^{1 / \beta} d s>\frac{(n-2) !}{e} .
$$

Agarwal et al. in [2] extended the Riccati transformation obtaining as new oscillation criterion for (2.1), the following condition:

$$
\limsup _{t \rightarrow \infty} t^{\beta(n-1)} \int_{t}^{\infty} q(s) d s>((n-1) !)^{\beta} .
$$

In [9] the authors studied the oscillatory behaviour of (2.1) for $\beta=1$. Using the Riccati transformation, they show that (2.1) is oscillatory if there exists a function $\mu \in C^{1}\left(\left[t_{0}, \infty\right),(0, \infty)\right)$, such that

$$
\int_{t_{0}}^{\infty}\left(\mu(s) q(s)-\frac{(n-2) !\left(\mu^{\prime}(s)\right)^{2}}{2^{3-2 n} s^{n-2} \mu(s)}\right) d s=\infty .
$$

We apply the previous results to the equation

$$
w^{(4)}(t)+\frac{q_{0}}{t^{4}} w(3 t)=0 . t \geq 1,
$$

(1) Applying the condition (2.2) contained in [1], we get

$$
q_{0}>13.6 \text {. }
$$


(2) Applying condition (2.3) contained in [2], we get

$$
q_{0}>18 \text {. }
$$

(3) Applying condition (2.4) contained in [9], we get

$$
q_{0}>576 .
$$

From the above results, we can infer that the results in [2] improve the results contained in [9]. Moreover, the results in [1] improve the results [2,9].

Thus, the motivation of this paper is complement and enrich the results in [1,2,9].

The key idea of our approach is to conduct a comparison with first order equations whose oscillatory behaviours are already known, using Riccati transformation technique.

The rest of the paper is organized as follows. Section 3 is devoted to the main results of the paper. We present our investigations for equation (1.1). Meanwhile, a relevant result on the existence of oscillatory oscillatory behaviour of solutions for first order differential equations is stated. The proofs rely on some mathematical inequalities and lemmas which are given for the sake of completeness. In Section 4 , we provide two examples with specific parameters to illustrate the applicability of our theorems. The paper ends with some concluding remarks.

The following lemmas will be very useful in the sequel:

Lemma 1 ([16]). If the function $w$ satisfies $w^{(i)}(t)>0, i=0,1, \ldots, n$, and $w^{(n+1)}(t)<0$, then

$$
\frac{w(t)}{t^{n} / n !} \geq \frac{w^{\prime}(t)}{t^{n-1} /(n-1) !} .
$$

Lemma 2 ([25]). Suppose that $w \in C^{n}\left(\left[t_{0}, \infty\right),(0, \infty)\right), w^{(n)}$ is of a fixed sign on $\left[t_{0}, \infty\right), w^{(n)}$ not identically zero and there exists a $t_{1} \geq t_{0}$ such that

$$
w^{(n-1)}(t) w^{(n)}(t) \leq 0,
$$

for all $t \geq t_{1}$. If we have $\lim _{t \rightarrow \infty} w(t) \neq 0$, then there exists $t_{\theta} \geq t_{1}$ such that

$$
w(t) \geq \frac{\theta}{(n-1) !} t^{n-1}\left|w^{(n-1)}(t)\right|,
$$

for every $\theta \in(0,1)$ and $t \geq t_{\theta}$.

Lemma 3 ([3]). Let $\beta$ be a ratio of two odd numbers, $V>0$ and $U$ are constants. Then

$$
U x-V x^{(\beta+1) / \beta} \leq \frac{\beta^{\beta}}{(\beta+1)^{\beta+1}} \frac{U^{\beta+1}}{V^{\beta}}, V>0 .
$$


Lemma 4 ([15]). Assume that $w$ is an eventually positive solution of (1.1). Then, there exist two possible cases:

$\left(\mathbf{S}_{1}\right) \quad w(t)>0, w^{\prime}(t)>0, w^{\prime \prime}(t)>0, w^{(n-1)}(t)>0, w^{(n)}(t)<0$,

$\left(\mathbf{S}_{2}\right) \quad w(t)>0, w^{(r)}(t)>0, w^{(r+1)}(t)<0$ for all odd integer $r \in\{1,3, \ldots, n-3\}, w^{(n-1)}(t)>0, w^{(n)}(t)<0$,

for $t \geq t_{1}$, where $t_{1} \geq t_{0}$ is sufficiently large.

\section{OSCILLATION CRITERIA}

In the following theorem, we compare the oscillatory behaviour of (1.1) with suitable first-order differential equations.

Theorem 1. Assume that (1) holds. If the differential equations

$$
x^{\prime}(t)+k \sum_{i=1}^{j} q_{i}(t)\left(\frac{\theta t^{n-2}}{(n-2) ! a^{1 / \beta}(t)}\right)^{\beta} x(\eta(t))=0
$$

and

$$
z^{\prime}(t)+z(t) \frac{t}{(n-4) !} \int_{t}^{\infty}(\varsigma-t)^{n-4}\left(\frac{k}{a(\varsigma)} \int_{\varsigma}^{\infty} \sum_{i=1}^{j} q_{i}(s) \mathrm{d} s\right)^{1 / \beta} \mathrm{d} \varsigma=0
$$

are oscillatory, then every solution of (1.1) is oscillatory.

Proof. On the contrary, let us assume that $w$ is a positive solution of (1.1). Then, we can suppose that $w(t)$ and $w\left(\eta_{i}(t)\right)$ are positive for all $t \geq t_{1}$ sufficiently large. From Lemma 4, we have two possible cases $\left(\mathbf{S}_{1}\right)$ and $\left(\mathbf{S}_{2}\right)$.

Let us assume that $\left(\mathbf{S}_{1}\right)$ holds. From Lemma 2, we get

$$
w(t) \geq \frac{\theta t^{n-2}}{(n-2) ! a^{1 / \beta}(t)}\left(a^{1 / \beta}(t) w^{(n-1)}(t)\right),
$$

for every $\theta \in(0,1)$ and for all large $t$. Thus, if we set

$$
x(t)=a(t)\left(w^{(n-1)}(t)\right)^{\beta}>0,
$$

then we see that $\delta$ is a positive solution of the inequality

$$
x^{\prime}(t)+k \sum_{i=1}^{j} q_{i}(t)\left(\frac{\theta t^{n-2}}{(n-2) ! a^{1 / \beta}(t)}\right)^{\beta} x(\eta(t)) \leq 0 .
$$

From [19, Theorem 1], we conclude that the corresponding equation (3.1) also has a positive solution, which is a contradiction.

Let us assume that $\left(\mathbf{S}_{2}\right)$ holds. Integrating (1.1) from $t$ to $m$ and using $w^{\prime}(t)>0$, we obtain

$$
a(m)\left(w^{(n-1)}(m)\right)^{\beta}-a(t)\left(w^{(n-1)}(t)\right)^{\beta}=-\int_{t}^{m} \sum_{i=1}^{j} q_{i}(s) g\left(w\left(\eta_{i}(s)\right)\right) d s .
$$


By virtue of $w^{\prime}(t)>0$ and $\eta_{i}(t) \geq t$, we get

$$
a(m)\left(w^{(n-1)}(m)\right)^{\beta}-a(t)\left(w^{(n-1)}(t)\right)^{\beta} \leq-k w^{\beta}(t) \int_{t}^{m} \sum_{i=1}^{j} q_{i}(s) d s .
$$

Letting $m \rightarrow \infty$, we see that

$$
a(t)\left(w^{(n-1)}(t)\right)^{\beta} \geq k w^{\beta}(t) \int_{t}^{\infty} \sum_{i=1}^{j} q_{i}(s) \mathrm{d} s
$$

and so

$$
w^{(n-1)}(t) \geq w(t)\left(\frac{k}{a(t)} \int_{t}^{\infty} \sum_{i=1}^{j} q_{i}(s) \mathrm{d} s\right)^{1 / \beta} .
$$

Integrating again from $t$ to $\infty$ for a total of $n-4$ times, we get

$$
w^{\prime \prime}(t)+\frac{w(t)}{(n-4) !} \int_{t}^{\infty}(\varsigma-t)^{n-4}\left(\frac{k}{a(\varsigma)} \int_{\varsigma}^{\infty} \sum_{i=1}^{j} q_{i}(s) \mathrm{d} s\right)^{1 / \beta} \mathrm{d} \varsigma \leq 0 .
$$

Using Lemma 1, we get

$$
w(t) \geq t w^{\prime}(t)
$$

From (3.4) and (3.5), we get

$$
w^{\prime \prime}(t)+w^{\prime}(t) \frac{t}{(n-4) !} \int_{t}^{\infty}(\varsigma-t)^{n-4}\left(\frac{k}{a(\varsigma)} \int_{\varsigma}^{\infty} \sum_{i=1}^{j} q_{i}(s) \mathrm{d} s\right)^{1 / \beta} \mathrm{d} \varsigma \leq 0 .
$$

Thus, if we set

$$
z(t)=w^{\prime}(t)
$$

then we see that $\delta$ is a positive solution of the inequality

$$
z^{\prime}(t)+z(t) \frac{t}{(n-4) !} \int_{t}^{\infty}(\varsigma-t)^{n-4}\left(\frac{k}{a(\varsigma)} \int_{\varsigma}^{\infty} \sum_{i=1}^{j} q_{i}(s) \mathrm{d} s\right)^{1 / \beta} \mathrm{d} \varsigma \leq 0
$$

It is well known (see [19, Theorem 1]) that the corresponding equation (3.2) also has a positive solution, which is a contradiction. The proof is complete.

Corollary 1. Assume that (1) holds. If

$$
\liminf _{t \rightarrow \infty} \int_{\eta_{i}(t)}^{t} \sum_{i=1}^{j} q_{i}(s)\left(\frac{\theta t^{n-2}}{(n-2) ! a^{1 / \beta}(t)}\right)^{\beta} \mathrm{d} s>\frac{((n-1) !)^{\beta}}{\mathrm{e}}
$$

and

$$
\liminf _{t \rightarrow \infty} \int_{\eta_{i}(t)}^{t} \frac{s}{(n-4) !} \int_{t}^{\infty}(\varsigma-t)^{n-4}\left(\frac{k}{a(\varsigma)} \int_{\varsigma}^{\infty} \sum_{i=1}^{j} q_{i}(s) \mathrm{d} s\right)^{1 / \beta} \mathrm{d} \zeta \mathrm{d} s>\frac{1}{\mathrm{e}}
$$

are oscillatory, then every solution of (1.1) is oscillatory. 
Lemma 5. Assume that $w$ be an eventually positive solution of (1.1) and $\left(\mathbf{S}_{1}\right)$ holds. If

$$
\phi(t):=\mu(t)\left(\frac{a(t)\left(w^{(n-1)}(t)\right)^{\beta}}{w^{\beta}(t)}\right),
$$

where $\mu \in C^{1}\left(\left[t_{0}, \infty\right),(0, \infty)\right)$, then

$$
\phi^{\prime}(t) \leq \frac{\mu^{\prime}(t)}{\mu(t)} \phi(t)-k \mu(t) \sum_{i=1}^{j} q_{i}(t)-\frac{\beta \theta t^{n-2}}{(n-2) !(\mu(t) a(t))^{\frac{1}{\beta}}} \phi(t)^{\frac{\beta+1}{\beta}},
$$

for all $t>t_{1}$, where $t_{1}$ large enough.

Proof. Let $w$ is an eventually positive solution of (1.1) and let us assume that $\left(\mathbf{S}_{1}\right)$ holds. Thus, from Lemma 2, we get

$$
w^{\prime}(t) \geq \frac{\theta}{2} t^{n-2} w^{(n-1)}(t)
$$

for every $\theta \in(0,1)$ and for all large $t$. From (3.9), we see that $\phi(t)>0$ for $t \geq t_{1}$, and

$$
\begin{aligned}
\phi^{\prime}(t)= & \mu^{\prime}(t) \frac{a(t)\left(w^{(n-1)}(t)\right)^{\beta}}{w^{\beta}(t)}+\mu(t) \frac{\left(a\left(w^{(n-1)}\right)^{\beta}\right)^{\prime}(t)}{w^{\beta}(t)} \\
& -\beta \mu(t) \frac{w^{\beta-1}(t) w^{\prime}(t) a(t)\left(w^{(n-1)}(t)\right)^{\beta}}{w^{2 \beta}(t)} .
\end{aligned}
$$

Using (3.11) and (3.9), we obtain

$$
\begin{aligned}
\phi^{\prime}(t) \leq & \frac{\mu_{+}^{\prime}(t)}{\mu(t)} \phi(t)+\mu(t) \frac{\left(a(t)\left(w^{(n-1)}(t)\right)^{\beta}\right)^{\prime}}{w^{\beta}(t)} \\
& -\beta \mu(t) \frac{\theta}{(n-2) !} t^{n-2} \frac{a(t)\left(w^{(n-1)}(t)\right)^{\beta+1}}{w^{\beta+1}(t)} \\
\leq & \frac{\mu^{\prime}(t)}{\mu(t)} \phi(t)+\mu(t) \frac{\left(a(t)\left(w^{(n-1)}(t)\right)^{\beta}\right)^{\prime}}{w^{\beta}(t)} \\
& -\frac{\beta \theta t^{n-2}}{(n-2) !(\mu(t) a(t))^{\frac{1}{\beta}}} \phi(t)^{\frac{\beta+1}{\beta}} .
\end{aligned}
$$

From (1.1) and (3.12), we obtain

$$
\phi^{\prime}(t) \leq \frac{\mu^{\prime}(t)}{\mu(t)} \phi(t)-k \mu(t) \frac{\sum_{i=1}^{j} q_{i}(t) w^{\beta}\left(\eta_{i}(t)\right)}{w^{\beta}(t)}-\frac{\beta \theta t^{n-2}}{(n-2) !(\mu(t) a(t))^{\frac{1}{\beta}}} \phi(t)^{\frac{\beta+1}{\beta}} .
$$


Note that $w^{\prime}(t)>0$ and $\eta_{i}(t) \geq t$, thus, we find

$$
\phi^{\prime}(t) \leq \frac{\mu^{\prime}(t)}{\mu(t)} \phi(t)-k \mu(t) \sum_{i=1}^{j} q_{i}(t)-\frac{\beta \theta t^{n-2}}{(n-2) !(\mu(t) a(t))^{\frac{1}{\beta}}} \phi(t)^{\frac{\beta+1}{\beta}} .
$$

The proof is complete.

Lemma 6. Assume that $w$ be an eventually positive solution of (1.1) and $\left(\mathbf{S}_{2}\right)$ holds. If

$$
\delta(t):=\vartheta(t) \frac{w^{\prime}(t)}{w(t)} .
$$

where $\vartheta \in C^{1}\left(\left[t_{0}, \infty\right),(0, \infty)\right)$, then

$$
\delta^{\prime}(t) \leq \frac{\vartheta^{\prime}(t)}{\vartheta(t)} \delta(t)-\Phi(t)-\frac{1}{\vartheta(t)} \delta(t)^{2}
$$

for all $t>t_{1}$, where $t_{1}$ large enough and

$$
\Phi(t)=\frac{\vartheta(t)}{(n-4) !} \int_{t}^{\infty}(\varsigma-t)^{n-4}\left(\frac{k}{a(\varsigma)} \int_{\varsigma}^{\infty} \sum_{i=1}^{j} q_{i}(s) \mathrm{d} s\right)^{1 / \beta} \mathrm{d} \zeta .
$$

Proof. Let $w$ is an eventually positive solution of (1.1) and let us assume that $\left(\mathbf{S}_{2}\right)$ holds. From the definition of $\delta(t)$, we see that $\delta(t)>0$ for $t \geq t_{1}$. By differentiating, we find

$$
\delta^{\prime}(t)=\frac{\vartheta^{\prime}(t)}{\vartheta(t)} \delta(t)+\vartheta(t) \frac{w^{\prime \prime}(t)}{w(t)}-\frac{1}{\vartheta(t)} \delta(t)^{2}
$$

From (3.4) and (3.15), we obtain

$$
\delta^{\prime}(t) \leq \frac{\vartheta^{\prime}(t)}{\vartheta(t)} \delta(t)-\frac{\vartheta(t)}{(n-4) !} \int_{t}^{\infty}(\varsigma-t)^{n-4}\left(\frac{k}{a(\varsigma)} \int_{\varsigma}^{\infty} \sum_{i=1}^{j} q_{i}(s) \mathrm{d} s\right)^{1 / \beta} \mathrm{d} \varsigma-\frac{1}{\vartheta(t)} \delta(t)^{2}
$$

Thus,

$$
\delta^{\prime}(t) \leq \frac{\vartheta^{\prime}(t)}{\vartheta(t)} \delta(t)-\Phi(t)-\frac{1}{\vartheta(t)} \delta(t)^{2}
$$

The proof is complete.

In this theorem, we will establish an oscillation criterion for equation (1.1).

Theorem 2. Assume that there exist positive functions $\mu, \vartheta \in C^{1}\left(\left[t_{0}, \infty\right),(0, \infty)\right)$ such that

$$
\int_{t_{0}}^{\infty}\left(k \mu(s) \sum_{i=1}^{j} q_{i}(s)-\frac{((n-2) !)^{\beta} a(t)\left(\mu^{\prime}(t)\right)^{\beta+1}}{(\beta+1)^{\beta+1}\left(t^{n-2} \theta \mu(t)\right)^{\beta}}\right) d s=\infty,
$$

for some $\theta \in(0,1)$, and either

$$
\int_{t_{0}}^{\infty} \sum_{i=1}^{j} q_{i}(s) d s=\infty
$$


or

$$
\int_{t_{0}}^{\infty}\left(\Phi(s)-\frac{1}{4 \vartheta(s)}\left(\vartheta^{\prime}(s)\right)^{2}\right) \mathrm{d} s=\infty
$$

Then every solution of (1.1) is oscillatory.

Proof. Assume that $w$ is an eventually positive solution of (1.1). Then, we can suppose that $w(t)$ and $w\left(\eta_{i}(t)\right)$ are positive for all $t \geq t_{1}$ sufficiently large. From Lemma 4, we have two possible cases $\left(\mathbf{S}_{1}\right)$ and $\left(\mathbf{S}_{2}\right)$.

Assume that $\left(\mathbf{S}_{1}\right)$ holds. From Lemma 5, we get that (3.10) holds. Using Lemma 3 with

$$
U=\frac{\mu^{\prime}(t)}{\mu(t)}, \quad V=\frac{\beta \theta t^{n-2}}{(n-2) !(\mu(t) a(t))^{\frac{1}{\beta}}} \text { and } x=\phi(t),
$$

we get

$$
\frac{\mu^{\prime}(t)}{\mu(t)} \phi(t)-\frac{\beta \theta t^{n-2}}{(n-2) !(\mu(t) a(t))^{\frac{1}{\beta}}} \phi(t)^{\frac{\beta+1}{\beta}} \leq-\frac{((n-2) !)^{\beta} a(t)\left(\mu^{\prime}(t)\right)^{\beta+1}}{(\beta+1)^{\beta+1}\left(t^{n-2} \theta \mu(t)\right)^{\beta}} .
$$

From (3.10) and (3.19), we obtain

$$
\phi^{\prime}(t) \leq-k \mu(t) \sum_{i=1}^{j} q_{i}(t)+\frac{((n-2) !)^{\beta} a(t)\left(\mu^{\prime}(t)\right)^{\beta+1}}{(\beta+1)^{\beta+1}\left(t^{n-2} \theta \mu(t)\right)^{\beta}} .
$$

Integrating from $t_{1}$ to $t$, we get

$$
\int_{t_{1}}^{t}\left(k \mu(s) \sum_{i=1}^{j} q_{i}(s)-\frac{((n-2) !)^{\beta} a(t)\left(\mu^{\prime}(t)\right)^{\beta+1}}{(\beta+1)^{\beta+1}\left(t^{n-2} \theta \mu(t)\right)^{\beta}}\right) \mathrm{d} s \leq \phi\left(t_{1}\right),
$$

for every $\theta \in(0,1)$, which contradicts (3.16). Assume that $\left(\mathbf{S}_{2}\right)$ holds. Integrating (1.1) from $m$ to $t$, we conclude that

$$
-a(m)\left(w^{(n-1)}(m)\right)^{\beta}=-\int_{m}^{t} \sum_{i=1}^{j} q_{i}(s) g\left(w\left(\eta_{i}(s)\right)\right) d s .
$$

By virtue of $w^{\prime}(t)>0$ and $\eta_{i}(t) \geq t$, we get

$$
\int_{m}^{t} \sum_{i=1}^{j} q_{i}(s) d s \leq \frac{a(m)\left(w^{(n-1)}(m)\right)^{\beta}}{k w^{\beta}(m)}
$$

which contradicts (3.17).

From Lemma 6, we get that (3.14) holds. Using Lemma 3 with

$$
U=\vartheta^{\prime}(t) / \vartheta(t), V=1 / \vartheta(t), \beta=1 \text { and } x=\delta(t),
$$

we get

$$
\frac{\vartheta^{\prime}(t)}{\vartheta(t)} \delta(t)-\frac{1}{\vartheta(t)} \delta^{2}(t) \leq-\frac{1}{4 \vartheta(t)}\left(\vartheta^{\prime}(t)\right)^{2}
$$


From (3.14) and (3.20), we obtain

$$
\delta^{\prime}(t) \leq-\Phi(t)+\frac{1}{4 \vartheta(t)}\left(\vartheta^{\prime}(t)\right)^{2} .
$$

Integrating from $t_{1}$ to $t$, we get

$$
\int_{t_{1}}^{t}\left(\Phi(s)-\frac{1}{4 \vartheta(s)}\left(\vartheta^{\prime}(s)\right)^{2}\right) \mathrm{d} s \leq \delta\left(t_{1}\right),
$$

which contradicts (3.18). The proof is complete.

Putting $\mu(t)=t^{n-1}$ and $\vartheta(t)=t$ into Theorem 2, we get the following oscillation criterion:

Corollary 2. Let (1.1) hold. Assume that

$$
\int_{t_{0}}^{\infty}\left(s^{n-1} \sum_{i=1}^{j} q_{i}(s)-\frac{((n-2) !)^{\beta}(n-1)^{\beta+1} s^{-n \beta+n+\beta-2} a(s)}{(\beta+1)^{\beta+1} \theta^{\beta}}\right) \mathrm{d} s=\infty,
$$

or some $\theta \in(0,1)$. If (3.17) holds and

$$
\int_{t_{0}}^{\infty}\left(\frac{s}{(n-4) !} \int_{t}^{\infty}(\varsigma-t)^{n-4}\left(\frac{k}{a(\varsigma)} \int_{\varsigma}^{\infty} \sum_{i=1}^{j} q_{i}(s) \mathrm{d} s\right)^{1 / \beta} \mathrm{d} \varsigma-\frac{1}{4 s}\right) \mathrm{d} s=\infty,
$$

then every solution of (1.1) is oscillatory.

\section{EXAMPLES AND CONCLUDING REMARKS}

In this section we show two numerical examples as application of the theoretical findings discussed in the previous section. Finally, we list some perspectives for future works.

Example 1. Let us consider the differential equation

$$
\left(t\left(w^{\prime \prime \prime}(t)\right)\right)^{\prime}+\frac{d_{0}}{t^{3}} w(c t)=0, t \geq 1,
$$

where $c>0$ and $d_{0}>1$ is a constant. Note that $\beta=1, n=4, a(t)=t, q(t)=d_{0} / t^{3}$. If we set $k=1$, and $\mu(s)=s^{3}$,then

$$
\begin{aligned}
& \int_{t_{0}}^{\infty}\left(k \mu(s) \sum_{i=1}^{j} q_{i}(s)-\frac{((n-2) !)^{\beta} a(s)\left(\mu^{\prime}(s)\right)^{\beta+1}}{(\beta+1)^{\beta+1}\left(s^{n-2} \theta \mu(s)\right)^{\beta}}\right) \mathrm{d} s \\
& =\int_{t_{0}}^{\infty}\left(d_{0} \frac{s^{3}}{s^{3}}-\frac{3^{2} s^{5}}{2 \theta s^{5}}\right) \mathrm{d} s=\int_{t_{0}}^{\infty}\left(d_{0}-\frac{9}{2 \theta}\right) d s \\
& =\infty \text { if } d_{0}>\frac{9}{2 \theta},
\end{aligned}
$$


for some constant $\theta \in(0,1)$. Hence, by Theorem 2, every solution of equation (4.1) is oscillatory if

$$
d_{0}>\frac{9}{2 \theta} .
$$

Remark 1. Applying the condition (3.22) to the equation (2.5), we find

$$
q_{0}>4.5 \text {. }
$$

Therefore, our result improves the results contained in [1,2,9].

Example 2. Let us consider the differential equation

$$
w^{(4)}(t)+\frac{q_{0}}{t^{4}} w(2 t)=0, t \geq 1,
$$

where $q_{0}>0$ is a constant. Note that $\beta=1, n=4, a(t)=1, q(t)=q_{0} / t^{4}$ and $\eta(t)=2 t$. If we set $k=1$, then condition (3.16) becomes

$$
\begin{aligned}
& \int_{t_{0}}^{\infty}\left(s^{n-1} \sum_{i=1}^{j} q_{i}(s)-\frac{((n-2) !)^{\beta}(n-1)^{\beta+1} s^{-n \beta+n+\beta-2} a(s)}{(\beta+1)^{\beta+1} \theta^{\beta}}\right) \mathrm{d} s \\
& =\int_{t_{0}}^{\infty}\left(\frac{q_{0}}{s}-\frac{9}{2 \theta s}\right) d s=\left(q_{0}-\frac{9}{2 \theta}\right) \int_{t_{0}}^{\infty} \frac{1}{s} d s \\
& =\infty \text { if } q_{0}>4.55(\text { let } \theta=99 / 100)
\end{aligned}
$$

and condition (3.18) becomes

$$
\begin{aligned}
& \left(\frac{s}{(n-4) !} \int_{t}^{\infty}(\varsigma-t)^{n-4}\left(\frac{k}{a(\varsigma)} \int_{\varsigma}^{\infty} \sum_{i=1}^{j} q_{i}(s) \mathrm{d} s\right)^{1 / \beta} \mathrm{d} \varsigma-\frac{1}{4 s}\right) \mathrm{d} s \\
& =\int_{t_{0}}^{\infty}\left(\frac{q_{0}}{6 s}-\frac{1}{4 s}\right) \mathrm{d} s \\
& =\infty, \text { if } q_{0}>\frac{3}{2} .
\end{aligned}
$$

Therefore, from Corollary 2, every solution to the equation (4.2) is oscillatory if $q_{0}>4.55$.

Remark 2. Now we compare our result with the known related criteria for oscillation of the equation under consideration.

(1) Applying condition (2.2) in [1], we get

$$
q_{0}>25.5 \text {. }
$$

(2) Applying condition (2.3) in [2], we obtain

$$
q_{0}>18 \text {. }
$$


(3) Applying condition (2.4) in [9], we find

$$
q_{0}>1728 .
$$

Therefore, our result improves the results contained in [1,2,9].

\section{ACKNOWLEDGEMENT}

The authors express their gratitude to the referee for his/her careful reading of the manuscript.

\section{REFERENCES}

[1] R. P. Agarwal and S. R. Grace, "Oscillation theorems for certain functional differential equations of higher order," Math. Comput. Modelling, vol. 39, no. 9-10, pp. 1185-1194, 2004, doi: 10.1016/S0895-7177(04)90539-0. [Online]. Available: https://doi.org/10.1016/S0895-7177(04) 90539-0

[2] R. P. Agarwal, S. R. Grace, and D. O'Regan, "Oscillation criteria for certain $n$th order differential equations with deviating arguments," J. Math. Anal. Appl., vol. 262, no. 2, pp. 601-622, 2001, doi: 10.1006/jmaa.2001.7571. [Online]. Available: https://doi.org/10.1006/jmaa.2001.7571

[3] O. Bazighifan, E. M. Elabbasy, and O. Moaaz, "Oscillation of higher-order differential equations with distributed delay,” J. Inequal. Appl., pp. Paper No. 55, 9, 2019, doi: 10.1186/s13660-019-2003-0. [Online]. Available: https://doi.org/10.1186/s13660-019-2003-0

[4] O. Bazighifan, "Kamenev and Philos-types oscillation criteria for fourth-order neutral differential equations," Adv. Difference Equ., 2020, doi: 10.1186/s13662-020-02661-6. [Online]. Available: https://doi.org/10.1186/s13662-020-02661-6

[5] O. Bazighifan and C. Cesarano, "Some new oscillation criteria for second order neutral differential equations with delayed arguments," Mathematics, vol. 7, 07 2019, doi: 10.3390/math7070619.

[6] O. Bazighifan and H. Ramos, "On the asymptotic and oscillatory behavior of the solutions of a class of higher-order differential equations with middle term," Appl. Math. Lett., vol. 107, pp. 106431, 9, 2020, doi: 10.1016/j.aml.2020.106431. [Online]. Available: https://doi.org/10.1016/j.aml.2020.106431

[7] O. Bazighifan, M. Ruggieri, and A. Scapellato, "An improved criterion for the oscillation of fourth-order differential equations," Mathematics, vol. 8, 04 2020, doi: 10.3390/math8040610.

[8] C. Cesarano and O. Bazighifan, "Oscillation of fourth-order functional differential equations with distributed delay," Axioms, vol. 8, 05 2019, doi: 10.3390/axioms8020061.

[9] S. R. Grace and B. S. Lalli, "Oscillation theorems for nth order nonlinear differential equations with deviating arguments." Proc. Am. Math. Soc., vol. 90, pp. 65-70, 1984.

[10] S. R. Grace, J. Džurina, I. Jadlovská, and T. Li, "On the oscillation of fourth-order delay differential equations," Adv. Difference Equ., 2019, doi: 10.1186/s13662-019-2060-1. [Online]. Available: https://doi.org/10.1186/s13662-019-2060-1

[11] S. Grace and J. Graef, "Oscillatory behavior of second order nonlinear difference equations with a nonlinear nonpositive neutral term," Miskolc Math. Notes, vol. 20, no. 2, pp. 899-910, 2019, doi: 10.18514/MMN.2019.2731.

[12] I. Győri and G. Ladas, Oscillation theory of delay differential equations: with applications, ser. Oxford Mathematical Monographs. Oxford University Press, USA, 1992.

[13] J. Hale, Theory of functional differential equations, 2nd ed. Springer-Verlag, New YorkHeidelberg, 1977.

[14] T. Li, B. Baculíková, J. Džurina, and C. Zhang, "Oscillation of fourth-order neutral differential equations with p-Laplacian like operators." Bound. Value Probl., vol. 2014, p. 9, 2014, id/No 56. 
[15] O. Moaaz, J. Awrejcewicz, and O. Bazighifan, "A new approach in the study of oscillation criteria of even-order neutral differential equations," Mathematics, vol. 8, no. 2, 2020, doi: 10.3390/math8020197. [Online]. Available: https://www.mdpi.com/2227-7390/8/2/197

[16] O. Moaaz, E. M. Elabbasy, and O. Bazighifan, "On the asymptotic behavior of fourth-order functional differential equations.” Adv. Difference Equ., p. 13, 2017, id/No 261.

[17] O. Moaaz, E. M. Elabbasy, and A. Muhib, "Oscillation criteria for even-order neutral differential equations with distributed deviating arguments," Adv. Difference Equ., 2019, doi: 10.1186/s13662-019-2240-z. [Online]. Available: https://doi.org/10.1186/s13662-019-2240-z

[18] Z. Nehari, "Oscillation criteria for second-order linear differential equations," Trans. Am. Math. Soc., vol. 85, 07 1957, doi: 10.2307/1992939.

[19] C. G. Philos, "On the existence of nonoscillatory solutions tending to zero at infinity for differential equations with positive delays." Arch. Math., vol. 36, pp. 168-177, 1981.

[20] M. A. Ragusa, "Elliptic boundary value problem in vanishing mean oscillation hypothesis," Comment. Math. Univ. Carolin., vol. 40, no. 4, pp. 651-663, 1999.

[21] M. A. Ragusa, "On weak solutions of ultraparabolic equations," Nonlinear Anal.-Theory Methods Appl., vol. 47, 2001, doi: 10.1016/s0362-546x(01)00195-x.

[22] M. A. Ragusa, "Homogeneous herz spaces and regularity results," Nonlinear Anal.-Theory Methods Appl., vol. 71, 2009, doi: 10.1016/j.na.2009.02.075.

[23] P. Řehák, "How the constants in Hille-Nehari theorems depend on time scales," Adv. Difference Equ., 2006, doi: 10.1155/ade/2006/31283. [Online]. Available: https://doi.org/10.1155/ade/2006/ 31283

[24] C. Zhang, R. P. Agarwal, M. Bohner, and T. Li, "New results for oscillatory behavior of even-order half-linear delay differential equations," Appl. Math. Lett., vol. 26, no. 2, pp. 179-183, 2013, doi: 10.1016/j.aml.2012.08.004. [Online]. Available: https://doi.org/10.1016/j.aml.2012.08.004

[25] C. Zhang, T. Li, B. Sun, and E. Thandapani, "On the oscillation of higher-order half-linear delay differential equations," Appl. Math. Lett., vol. 24, no. 9, pp. 1618-1621, 2011, doi: 10.1016/j.aml.2011.04.015. [Online]. Available: https://doi.org/10.1016/j.aml.2011.04.015

Authors' addresses

Omar Bazighifan

Department of Mathematics, Faculty of Science, Hadhramout University, 50512 Hadhramout, Yemen

Department of Mathematics, Faculty of Education, Seiyun University, 50512 Seiyun, Yemen

E-mail address: 0 .bazighifanegmail.com

Andrea Scapellato

Università degli Studi di Catania, Dipartimento di Matematica e Informatica, Viale Andrea Doria 6 95125 Catania, Italy

E-mail address: andrea.scapellato@unict.it 